\title{
AUTOMATIC GEOMETRIC AND PHOTOMETRIC CALIBRATION FOR TILING MULTIPLE PROJECTORS WITH A PAN-TILT-ZOOM CAMERA
}

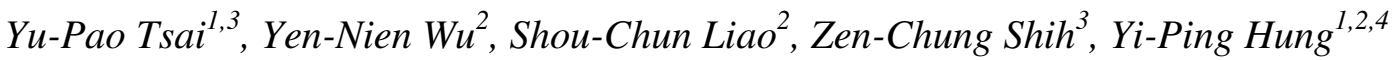 \\ ${ }^{1}$ Institute of Information Science, Academia Sinica, Taiwan. \\ ${ }^{2}$ Department of Computer Science and Information Engineering, National Taiwan University, Taiwan. \\ ${ }^{3}$ Department of Computer and Information Science, National Chiao Tung University, Taiwan. \\ ${ }^{4}$ Institute of Networking and Multimedia, National Taiwan University, Taiwan. \\ Email: hung@csie.ntu.edu.tw
}

\begin{abstract}
Research on creating a large, high-resolution, low-cost display system has become increasingly important due to the growing desire in many fields for bigger and better displays. The goal of this research is to build a seamless large-scale display system by tiling multiple projectors with the help of a pan-tilt-zoom camera. In order to achieve this goal, we took two tasks into consideration: geometric calibration and photometric calibration. Compared to the previous work, our method for geometric calibration is more accurate, thanks to the much higher resolution images acquired by combining several zoom-in images and doing lens correction for the camera at first. For photometric calibration, we achieve color uniformity among projectors by utilizing the same PTZ camera, which is calibrated once with a colorimeter in a factory. Furthermore, we adopted the technique of producing a high dynamic range image from several images of different exposures to increase the measurement accuracy of the camera. In our experiments, the average error of photometric measurement achieved by our method is less than the mean perceptibility tolerance. Our method has great potential for many applications that require large and high-resolution displays.
\end{abstract}

\section{INTRODUCTION}

Research in building large, high-resolution displays has become increasingly popular recently due to the constant yearn for bigger and better displays in the entertainment, industrial, and scientific worlds [5][10]. An approach utilizing multiple projectors has attracted a great deal of interest. This research aims to create large, high-resolution displays by presenting improved methods of creating a "projector mosaic"[9] which is a collection of projected images combined to form one large display.
This paper takes into account not only the quality of the outcome but the affordability of the equipment. In the past, most relative research uses a fixed-lens camera to do geometric calibration [2][6][8][10]. However, their methods require that the entire projected area be completely visible by the fixed-lens cameras. The calibration may further degrade the accuracy of the measurements when the projected area becomes large. In order to increase calibration accuracy, this paper proposes a technique to increase the measurement resolution by combining several zoom-in images acquired by a pan-tilt-zoom camera. The similar technique is adopted by Chen et al. [1]. However, we identify the feature points using coding technique with color patterns (figure 1(b)) so that we can measure the feature points with a higher zoom-in factor. Therefore, our method can achieve better accuracy. Notice that the camera is calibrated for lens distortion at first to reduce the measurement error. In addition, previously the color variation across a multi-projector display was calibrated by using an expensive device known as the spectroradiometer[7]. But in this paper, the same results are achieved with a comparatively low-cost camera with the technique of producing a high dynamic range image from several images of different exposures. The camera captures sub-sampled color images and uses the information to calibrate the colors between the multiple projectors to achieve color uniformity across the display. Finally the blending algorithm described proposes to assign the weights of the pixels near the edge to zero and the pixels in the nonoverlapped region to one. This feathering method thus relies on the distance between the edge and the pixels and incorporates the information obtained by the geometric and photometric calibration method.

In the remaining part of this paper, we will describe our method to deal with geometry misalignment in Section 2, and photometry variance in Section3. Next, we adopt intensity blending approach to reduce the light in the overlapping region. Finally some results and conclusion will be given. 


\section{GEOMETRIC CALIBRATION}

In geometric calibration, we have to obtain the homographies between the camera, projectors, and display screen by establishing feature correspondences between coordinate frames.

To calculate the homography between the camera and each projector, vertical lines and horizontal lines are respectively displayed onto the screen from the projector and detected in the image captured by the camera. Figure 1(a) shows the patterns. Each intersection of vertical and horizontal line defines a feature point. After obtaining the positions of feature points in the image, we can calculate the camera-toprojector homography by using the least-squares approach since the positions of the feature points in the projector coordinate are known. Rather than using a single view, we use multiple zoomed views to increase measurement resolution and to find out feature points more accurately. Figure 2 illustrates the process of geometric calibration using a pan-tilt-zoom camera.

The second task of geometric calibration is to calculate the homography between the camera and the display screen so that we can perform keystone correction to make the final projected image a rectangle. To indicate what orientation is desired, we put a rectangle pattern board in front of the screen in order to detect the orientation of the pattern board. Based on the detected orientation, our system automatically finds the largest rectangle that is the same orientation of the pattern board and inside the union of all the projected areas. Therefore, we can adjust the pattern board to decide the orientation of the final display area we want.

Since the homography relationship between the camera and the screen has already been calculated, as is the relationships between the projectors and the camera, the relationships between the projectors and the screen can be calculated. Knowing which projectors are projecting which pixels, this information can be used in the intensity blending module to calculate the weight intensity for each pixel of each projector.

\section{PHOTOMETRIC CALIBRATION}

To facilitate color balancing on a multi-projector display, we need to measure the color characteristics of the projectors. Traditionally, a sophisticated mechanical device such as colorimeter or spectroradiometer is utilized. In this paper, we use a comparatively low-cost camera to achieve the same results. Before using the camera, we need to do in-factory camera calibration to construct the color mapping between a colorimeter and the camera. Once the calibration is completed, we use the camera to find a common color gamut among the projectors. Further, we use a gamut matching algorithm to map the color gamut we want to display to the common color gamut. The details will be described in section 3.2.

In order to increase measurement accuracy of the camera, we adopt the method proposed by Debevec and Malik [3], which produces a high dynamic range image from several images of different exposures. Thus, in photometric calibration module, we use the camera to capture the projected color with different exposures and calculate its relative radiance value using the response function of the camera.

\subsection{In-factory Camera Calibration}

To construct the mapping between a colorimeter and the camera, we project some color samples onto the display screen. At the same time, we use a colorimeter, i.e., Photo Research PR650, to measure the projected color, and record its CIE-XYZ tristimulus value. Furthermore, we use the camera to obtain its relative radiance value. After measuring all color samples, we can construct a mapping between the CIE-XYZ tristimulus values and the relative radiance values of the sample colors. We denote the mapping as $M_{\mathrm{rad}}^{\mathrm{XYZ}}$. The mapping will be used in in-field calibration to transform the measured radiance value into the device independent color space CIE-XYZ [4][11].

\subsection{In-field Calibration}

Once the camera is calibrated, we can use it to measure the color gamut of each projector and then map the color gamut we want to display into the common color gamut which is the intersection of color gamuts of the projectors and is the range of colors displayable on all the projectors. Figure 3 illustrates the process of in-field calibration. To measure the color gamut of each projector, we project some sampled colors, and measure the radiance values of projected colors using the camera. Next, we translate the measured radiance values into CIE-XYZ color space using the mapping we constructed from in-factory calibration. Therefore, we can construct the mapping, $M_{P i}^{X Y Z}$, between the rgb values and CIE-XYZ tristimulus values for each projector $P_{i}$. Here, the colors are sampled on the boundary surface of the RGB cube, so that we can measure the surface boundary of the color gamut. After obtaining the color gamut $G_{i}$ of each projector $P_{i}$ and the color gamut $G_{s}$ of CIE-RGB, the color matching will applied to map the colors which will be displayed in our system to the common gamut. Given an input color $\left(X_{I}, Y_{I}, Z_{I}\right)$ in CIE-XYZ color space, we can obtain a output color $\left(X_{O}, Y_{O}, Z_{O}\right)$, which is inside the common gamut, after color matching. Finally, the value $\left(X_{O}, Y_{O}, Z_{O}\right)$ is transform to value $\left(r_{i}, g_{i}, b_{i}\right)$ using the mapping $M_{P i}^{X Y Z}$, and the color $\left(r_{i}, g_{i}, b_{i}\right)$ is displayed by projector $P_{i}$ so that the projected colors by all the projectors are the same. 
For color matching, we adopt a non-linear compression algorithm to convert the color gamut $G_{s}$ into the common gamut $G_{c}$. We perform our algorithm in CIE-La*b* color space, because it is a uniform color space [4][11]. In addition, we would like to preserve hue in color matching process, so we define a transformation from CIE-La*b* color space to a spherical coordinate $(p, t, \rho)$ which is centered at $(50,0,0)$ in CIE-La*b*, as shown in (1). Notice that if two colors have the same $p$ and $t$ values, they will be the same hue.

$$
p=\tan ^{-1}\left(\frac{a}{b}\right), t=\tan ^{-1}\left(\frac{L-50}{\sqrt{a^{2}+b^{2}}}\right), \rho=\sqrt{a^{2}+b^{2}+(L-50)^{2}}
$$

The process of the non-linear compression algorithm is following. First, we find out the common gamut $G_{c}$ which is the intersection of color gamuts of all the projectors. Second, we transfer the value $\left(X_{I}, Y_{I}, Z_{I}\right)$ to the value $\left(L_{I}, a_{I}, b_{I}\right)$, and then transfer it to the value $\left(p_{I}, t_{I}, \rho_{I}\right)$. Assume $\rho_{s}$ be the maximum $\rho$ value of color gamut $G_{s}$ with the angle $p_{I}$ and $t_{l}$, and $\rho_{c}$ be the maximum $\rho$ value of the common color gamut $G_{c}$ with the angle $p_{I}$ and $t_{I}$. In addition, we let $\rho_{n}$ be equal to $0.9^{*} \rho_{c}$. If $\rho_{I}$ is smaller than $\rho_{n}$, we assign $\left(X_{I}, Y_{I}, Z_{I}\right)$ to $\left(X_{O}, Y_{O}, Z_{O}\right)$. If it is not, the $\rho_{I}$ shrinks to $\rho_{I}$ ' by the equation (2).

$$
\rho_{I}^{\prime}=\frac{\left(\rho_{I}-\rho_{n}\right) *\left(\rho_{c}-\rho_{n}\right)}{\left(\rho_{s}-\rho_{n}\right)}+\rho_{n}
$$

After we get the new value of $\rho_{I}$, we can translate $\left(p_{I}, t_{I}, \rho_{I}{ }^{\prime}\right)$ back to CIE-XYZ color space, and assign it to $\left(X_{O}, Y_{O}, Z_{O}\right)$.

\section{INTENSITY BLENDING}

When two projections overlap, the overlapped region has twice the intensity as the non-overlapped regions. This overlapped region is very noticeable and can thus distract an audience from watching the picture, and instead attract their attention towards this bright region. Thus, in order to seamlessly merge the images without any obvious overlapped region, an intensity blending technique is used to smoothly combine the images into one large picture. This technique, known as feathering or cross-fading, calculates an intensity weight for each pixel of the projector image. The intensity weights of the two images in the overlapped regions add up to the same intensity as the non-overlapped region. The assignment of intensity weights to each pixel is also called a mask. This algorithm assigns the intensity weight near the edges to near zero and the weight increases for pixels far from the edge. The weight for the pixels in the non-overlapped regions is clearly one. This means that the light intensity starts out at one in the overlapped region and as it approaches the overlapped region, it slowly starts decreasing until it reaches the edge where the light intensity becomes zero.
Our display system consists of Polyvision DLP projectors and a Canon VC-C4 pan-tilt-zoom camera. For photometric calibration, we use 127 sampled colors to perform in-factory calibration. To evaluate the measurement error caused by using a camera to substitute a colorimeter, we project a randomly sampled color and get measurements by both the camera and colorimeter, and calculate the color difference $\Delta E^{*}{ }_{a b}$. The error was also calculated. We iteratively repeated the test 102 times. The average value of $\Delta E^{*}{ }_{a b}$ is 1.4380 , the maximum value is 6.8552 , and the minimum value is 0.021 . Observing these values, we can find that the average value is less than the mean perceptibility tolerance value of 3 and the maximum value is also less than the maximum perceptibility tolerance value of 12 [4]. Figure 4 shows an example of displaying an image in our display system. The input image is warped using the information obtained in geometric calibration module for each projector, and then the intensity of each pixel is calculated using the alpha value obtained in the intensity blending module. Next, the color matching is performed using the information obtained in photometric calibration module. Finally, the generated images are projected onto the display screen to produce a seamless result, as shown in Figure 4 (e).

\section{CONCLUSION}

In this paper, we propose an approach to construct a large seamless display by integrating multiple projectors with the help of a pan-tilt-zoom camera. In geometric calibration, we developed a technique to increase the measurement resolution by combining several zoom-in images acquired by a pan-tilt-zoom camera and doing lens correction for the camera at first. In the photometric calibration, we use a lowcost video camera, which is calibrated with colorimeter once in a factory, to measure the color response of the projector, and then adopt a color gamut compression algorithm to do color gamut mapping. Here, by utilizing the method of generating high dynamic range images, we developed a method to increase measurement accuracy. In our experiments, the average error of photometric measurement error achieved by our method is less than the mean perceptibility tolerance. Our method has great potential for many applications that require large and high-resolution displays, such as scientific visualizations and visual display walls.

\section{ACKNOWLEDGEMENTS}

This work was partially supported by the National Science Council, Taiwan through grant NSC92-2622-E-002-027CC3. We would also like to thank EeRise Co. for their supports.

\section{EXPERIMENTAL RESULTS}




\section{REFERENCE}

[1] H. Chen, R. Sukthankar, G. Wallace, T.-J. Cham, "Calibrating Scalable Multi-Projector Displays Using Camera Homography Trees," CVPR Technical Sketch, 2001.

[2] Y. Chen, D. W. Clark, A. Finkelstein, T. C. Housel, and K. Li, "Automatic Alignment of High-Resolution Multi-Projector Displays Using An Un-Calibrated Camera," Proc. of IEEE Visualization, 2000, pp. 125130.

[3] P. Debevec and J. Malik, "Recovering High Dynamic Range Radiance Maps from Photographs," Proc. ACM SIGGRAPH, pp. 369-378, 1997.

[4] P. Green and LW. MacDonald, eds, Color Engineering: Achieving Device Independent Colour, John Wiley \& Sons, England, in press, 2002.

[5] M. Hereld, I. Judson, and R. Stevens, "Introduction to Building Projection-based Tiled Display Systems," IEEE Computer Graphics and Applications, July/August 2000, pp. 22-28.

[6] K. Li, H. Chen, Y. Chen, D. W. Clark, P. Cook, S. Damianakis, G. Essl, A. Finkelstein, T. Funkhouser, T. Housel, A. Klein, Z.Liu, E. Praum, R. Samanta, B. Shedd, J. P. Singh, G. Tzanetakis, and J. Zheng, "Building and Using a Scalable Display Wall System," IEEE Computer Graphics and Applications, July/August 2000, pp. 29-37.

[7] A. Majumder, Z. He, H. Towles, and G. Welch, "Achieving Color Uniformity Across Multi-Projector Displays", Proc. of IEEE Visualization, 2000, pp. 117124.

[8] R. Raskar, M. S. Brown, R. Yang, W.-C. Chen, G. Welch, H. Towles, B. Seales, and H. Fuch, "MultiProjector Displays Using Camera-Based Registration," Proc. of IEEE Visualization, 1999, pp. 161-68.

[9] R. Raskar, J. V. Baar, and J. X. Chai, "A Low-Cost Project Mosaic with Fast Registration", Proc. of Asian Conference on Computer Vision (ACCV2002), 2002.

[10] R. Sukthankar, R. G. Stockton, and M. D. Mullin, "Smarter Presentations: Exploiting Homography in Camera-Projector Systems," Proc. of ICCV 2001, 2001, pp.

[11] G. Wyszecki, W.S. Stiles, "Color Science: Concepts and Methods, Quantitative Data and Formulae," Second Edition, Wiley, 1982.

[12] M. Brown, A. Majumder, and R. Yang, "Camera-Based Calibration Techniques for Seamless Multiprojector Displays," IEEE Transactions on Visualization and Computer Graphics, Vol. 11, No. 2, March-April, 2005.

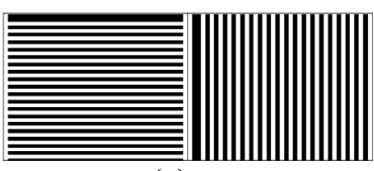

(a)

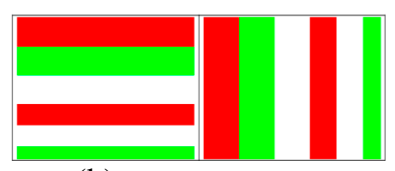

(b)
Figure 1. Feature patterns. (a) horizontal and vertical lines.

(b) color patterns used to identify the feature points.

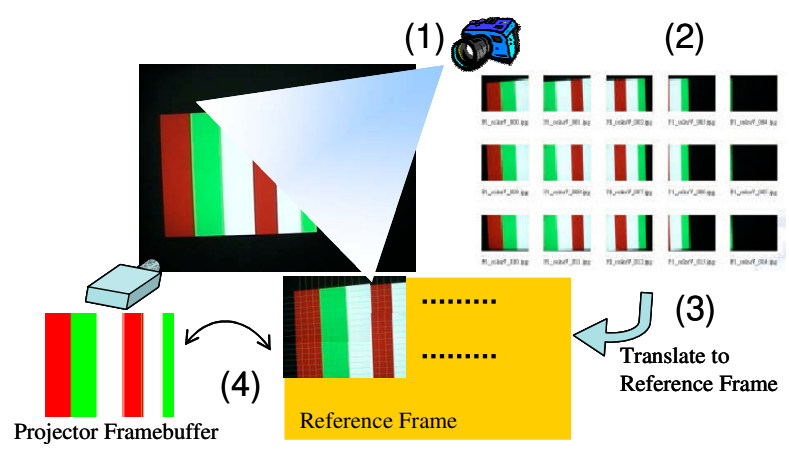

Figure 2. An illustration of geometric calibration

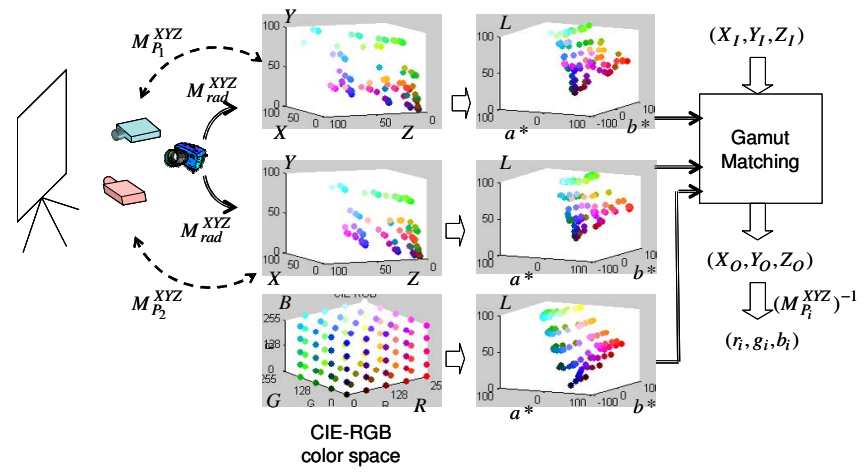

Figure 3. An illustration of in-field calibration.

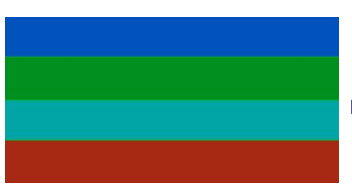

(a) source image

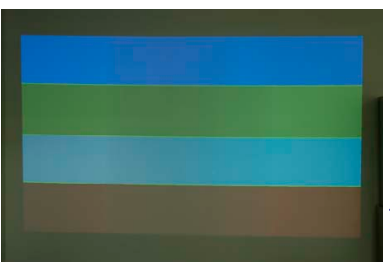

(e) final result

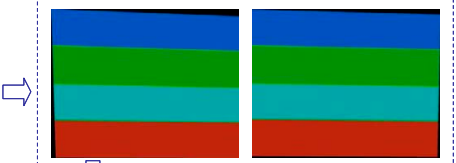

凸 (b) Warped images

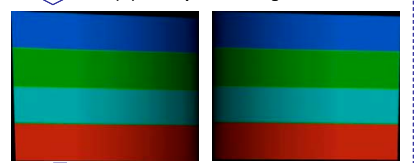

凸(c) Intensity blended images

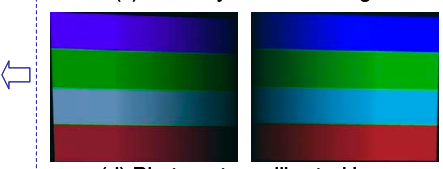

(d) Photometry calibrated images
Figure 4. An example of displaying a color image 